\title{
An Approximation to Red Blood Cells with a Model of Three-Center-Combined Shells
}

\author{
Q.L.ZHAN ${ }^{1,2}$, R.J.ZHANG ${ }^{1 *}$ \\ ${ }^{1}$ Institute of Applied Mechanics, Tongji University, Shanghai 200092, China \\ ${ }^{2}$ Department of Bridge Engineering, State Key Laboratory for Disaster Reduction in Civil Engineering, Tongji University \\ *Corresponding author zhangrj@tongji.edu.cn \\ Tel.:+86-21-65983267; fax: +86-21-65983267 \\ Accepted: April 5, 2015.
}

\begin{abstract}
Red blood cells present a biconcave shape and bear an inner pressure (osmotic pressure) when they are in the static state. In this paper, a model of "three-centre-combined shells", which consists of two spherical shells and a toroidal shell, is employed to describe the geometric shape of red blood cells. Surface area and volume of the combined shells model are very close to those measured from experiment. The stress distribution in the cell membrane is formulized as a closed form according to the Novozhilov's theory of the three-centre-combined shells. Calculating results in terms of Novozhilov's formula give a good agreement with the numerical results given by ABAQUS when using actual measurements. It is concluded that the combined shells model can well approximate to the biconcave structure of red blood cells. In addition, stress calculation shows that the membrane of biconcave red blood cells can carry bending moments, and the moments reach a maximum value in the vicinity of joint line of the spherical shell and the toroidal shell in the combined shells model.
\end{abstract}

Keywords: Shells, Combined shells, Red blood cells,Osmosis experiment

\section{Introduction}

Red blood cells tend to deform easily. They present biconcave shape when at rest and display various different shapes when flow. For example, they become extremely elongated and freely bent when flow into the micro vascular. The average life span of a red blood cell is 120 days. After expansion to sphere, it is ultimately devoid in the spleen [1].

Red blood cells usually bear an inner pressure. The amplitude of the inner pressure can be adjusted by the bidirectional permeability mechanism, and the inner pressure is also referred to as osmotic pressure. During osmosis experiments, when the red blood cells present a biconcave shape, the osmotic pressure is lower and there is less fluid in the cell. When the curvature of red blood cells is positive everywhere (gibbous), the osmotic pressure is higher and there is more fluid in the cell correspondingly.

When the shape of red blood cells is biconcave, there are two areas on the cell surface, which have positive and negative Gaussian curvatures, respectively. The joint of the two areas is called turning line or transition line. Thin shell theory indicates that there exist bending moments in the vicinity of the transition line. Therefore the cell membrane can sustain moments and behaves as a "real membrane" when the shape of red blood cells is biconcave. However, when the cell is spherical, its membrane is incapable of carrying any bending moment, and only provides surface tensions. In this situation it behaves as an "ideal membrane". As an ideal membrane the red blood cells can change their shapes arbitrarily when moving in a blood vessel. The concepts of "real" and "ideal" membrane described above are given by Libai [2].

Thus, it can be inferred that the cell membrane of living body can actively adjust the tensions of its different areas or the stress distribution in it to change itself into or "real" or "ideal" membrane and finally change its shape.

The transformation of the shape of red blood cells has been discussed earlier, for example, in the paper by Beck [3].

A few models have been developed to analyze the mechanical performance of cell membranes. Fung [4] established a variable thickness shell model for red blood cells. Evans [5] proposed a single strain energy function consisting of two terms to simulate the constitutive relation of the membranes of red blood cells. Helfrich [6] proposed a theory of the elasticity of lipid bilayers and explained the biconcave-discoid shape under normal physiological condition. Zarda [7] computed a large elastic deformation of red blood cells on the basis of an assumed model includes the elasticity of the membrane under tensions in its own plane and the bending elasticity. Zhan, Zhang and Jiao [8] simulated numerically the instantaneous deformation of red blood cells from biconcave into spherical shape and found a critical osmotic pressure.

Therefore, it is important to determine the stress distribution in the membrane of red blood cells when the cells have biconcave shape. However, it is not understood yet how the membrane adjusts its microstructure to form a desired 
stress distribution. It can be imagined that the adjustment mechanism is very flexible, and allows an arbitrary transformation between the real and the ideal membrane.

It is found in this paper that the biconcave geometry of red blood cells can be approximated in terms of a model of three-center-combined shells. As for the three-center -combined shells, the analytical expressions of stresses and straines have been given by Novozhilov [9] in his monograph. Those expressions are directly employed in this paper to calculate the stress distribution in the membrane of biconcave red blood cells. For better reading, the derivation of those expressions are listed in appendix. Some errors in the monograph are modificated. In order to verify those expressions, the ABAQUS software is employed to make a parallel calculation. Measured data and experimental data are used in all the culculations. Within limits of the authors' knowledge, it is not found yet in any literature that the three-center-combined shells are used to model red blood cells.

\section{Three-center-combined shells}

Three-center-combined shells are an assembly of two spherical shells and a toroidal shell. The shells are symmetrical about their horizontal middle plane. The upper half of the shells is shown in Fig. 1, in which the dotted line represents a spherical shell with radius of $R$; the solid line represents a toroidal shell, whose radius is $r_{0}$ and the rotational radius of its centerline is $R_{0}$; the angle at the joint of two kinds of shells is $\theta_{0}$; the thickness of the shells is $h$. As can be seen that there are three centers for the half of the combined shells, Novozhilov [9] referred them to as "three-center-combined shells".

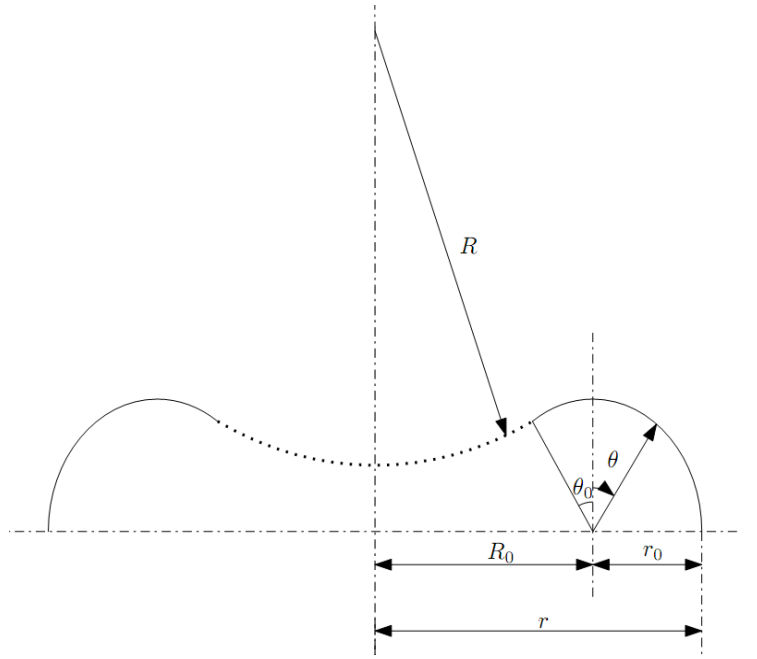

Fig. 1 Schematic diagram of three-center-combined shells

\section{Red blood cells with biconcave shape}

Evans and Fung [10] gave a formula to fit an average cross-sectional shape of red blood cells as follows:

$$
\begin{gathered}
\eta=0.5\left[1-\xi^{2}\right]^{\frac{1}{2}}\left(C_{0}+C_{1} \xi^{2}+C_{2} \xi^{4}\right) \\
X=3.91 \xi(\mu \mathrm{m}), Y=\eta(\mu \mathrm{m})
\end{gathered}
$$

where $\xi$ is in the range of $-1 \leq \xi \leq 1$, and $X$ and $Y$ are two coordinates; constants $C_{0}=0.207161, C_{1}=2.002558$ and $C_{2}=-1.122762$, respectively. Above formula is graphed in Fig. 2.

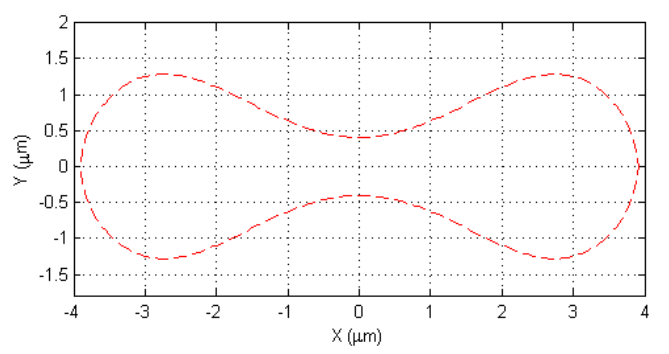

Fig. 2 Red blood cell fitting figure of measured data

\section{Model of three-center-combined shells}

By reason of symmetry, a quarter of the cell model is taken to be analyzed and shown in Fig. 3.

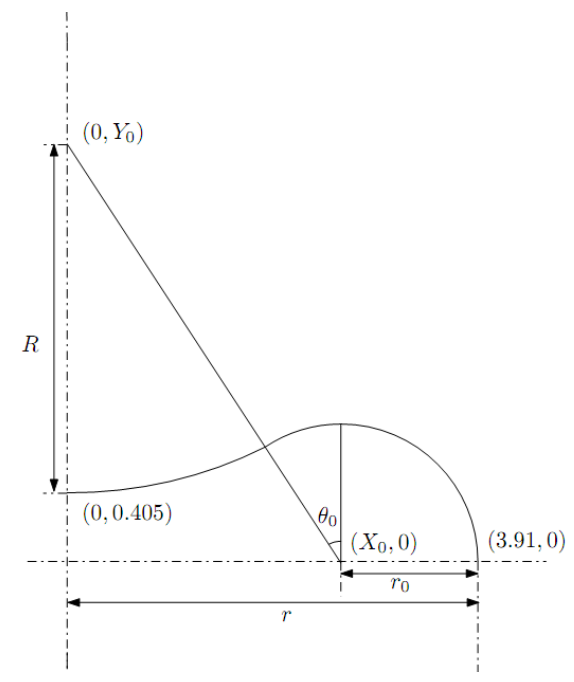

Fig. 3 Calculation model (units of $\mu \mathrm{m}$ )

where the center of spherical shells is located at $\left(0, Y_{0}\right)$ and the center of toroidal shells is at $\left(X_{0}, 0\right)$. According to the formula (1), it is easily determined that $r=3.91 \mu \mathrm{m}$ and $Y_{0}=0.405+R$. By adjusting the coordinates of the two centers and the radii of the two shells, it is finally determined that the radius of the spherical shells $r_{0}=1.2920 \mu \mathrm{m}$ and the associate abscissa is $X_{0}=2.6180 \mu \mathrm{m}$; the radius of the toroidal shells is $R=3.0150 \mu \mathrm{m}$ and the associate ordinate is $Y_{0}=3.4200 \mu \mathrm{m}$; the angle at the joint of the spherical and toroidal shells is $\theta_{0}=0.6533$. 


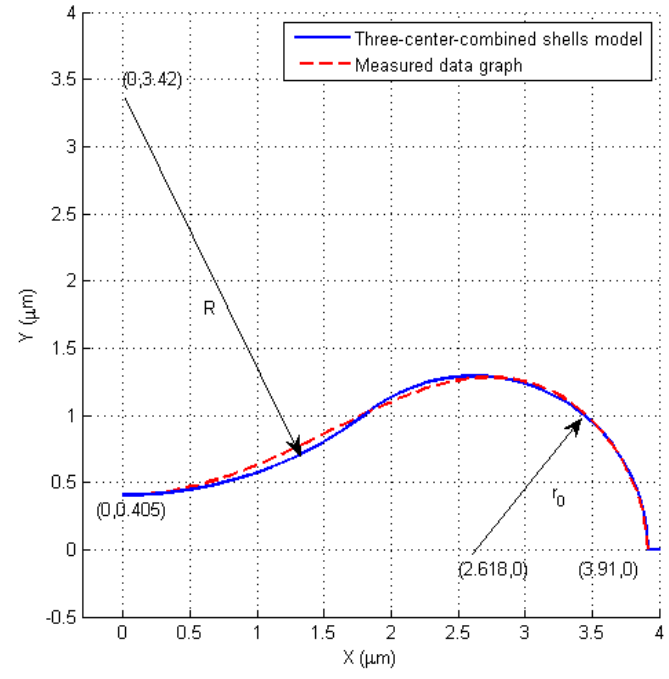

Fig. 4 Combined shells model and measured data for red blood cells

Two graphs depicted by using both the Eqn(1) and the combined shells model respectively are illustrated in Fig 4. In the figure, the red dashed line represents the graphical result of Eqn(1) for measured data, and the blue solid line is the graphical result of the combined shells model. It can be clearly seen from Fig. 4 that the combined shells model developed in this paper can well be used to simulate the actual shape of red blood cells.

Furthermore, the surface area and volume of red blood cells are calculated and compared with measured data to verify the precision of the new model. According to the aforementioned geometry of the three-center-combined shells, it is easily obtained that the surface area is $133.5066 \mu \mathrm{m}^{2}$ and the volume is $93.6963 \mu \mathrm{m}^{3}$. As for the measured value, Evans and Fung [10] and Tsang [11] used the interference method and received respectively $134.1 \mu \mathrm{m}^{2}$ and $129.95 \mu \mathrm{m}^{2}$ for the mean surface area and $94.1 \mu \mathrm{m}^{3}$ and $97.91 \mu \mathrm{m}^{3}$ for the mean volume, respectively. The results are given in Table 1. It is evident from this table that the surface area and volume of the combined shells model have a good agreement with the one of real cells.

Table 1 Comparison of surface area and volume of three-center-combined shells and measured data.

\begin{tabular}{lll}
\hline & Surface Area $\left(\mu m^{2}\right)$ & Volume $\left(\mu m^{3}\right)$ \\
\hline Three-center-combined shells & 133.5066 & 93.6963 \\
\hline Measured data by Evans & 134.1 & 94.1 \\
\hline Measured data by Tsang & 129.95 & 97.91 \\
\hline
\end{tabular}

It should be pointed out that some analytic expressions for cell volume and for the area of the membrane have been derived in the paper by Angelov and Mladenov [12].

\section{Stress distribution in the three-center-combined shells which are subjected to an internal pressure}

\subsection{Complex force resultants in shells of revolution}

For shells of revolution, there are three equations of equilibrium and three equations of compatibility. The two sets of equations have some kind of symmetry. Novozhilov [9] combined the six real equations into three complex equations by introducing three complex auxiliary functions. For a symmetrical deformation, the three complex equations can be further simplified to two which contain two complex auxiliary functions. Eliminating one of them gives only one complex equation with one complex auxiliary function. Novozhilov [9] called the complex auxiliary function $\tilde{T}$ "complex force resultant" which is defined as

$\tilde{T}=T_{2}-v T_{1}+(1+v) T_{1}-i \frac{M_{1}+M_{2}}{(1+v) c}$

where $i$ is imaginary unit; $v$ is Poisson's ratio; $c=h / \sqrt{12\left(1-v^{2}\right)} ; h$ is shell thickness; $T_{1}, T_{2}$, $M_{1}$ and $M_{2}$ are the force and moment resultants per unit length along two principal coordinates on shell surface.

The complex equation is

$\frac{d^{2} \tilde{T}}{d \theta^{2}}+\left[\left(2 \frac{R_{1}}{R_{2}}-1\right) \cot \theta-\frac{1}{R_{1}} \frac{d R_{1}}{d \theta}\right] \frac{d \tilde{T}}{d \theta}+i \frac{R_{1}^{2}}{R_{2} c} \tilde{T}=i \frac{R_{1}^{2}}{R_{2} c} F(\theta)$

For a uniform internal pressure, $F(\theta)$ can be written as

$F(\theta)=p R_{2}-\left(\frac{1}{R_{1}}-\frac{1}{R_{2}}\right) \frac{1}{\sin ^{2} \theta}\left(\tilde{C}_{1}+p \int_{\theta^{*}}^{\theta} R_{1} R_{2} \cos \theta \sin \theta d \theta\right)$

where $p$ is pressure on unit area (along exterior normal) and is constant for uniform internal pressure; $R_{1}$ is the radius of first principal curvature, that is the radius of curvature of meridian; $R_{2}$ is the radius of second principal curvature; $\theta^{*}$ is the starting angle of loads.

For symmetric deformation, $\tilde{T}$ is only the function of $\theta$ (the first principal coordinate). It is convenient to establish an arc coordinate $s$ on the meridian in this case, which gives that $d s=R_{1} d \theta$. Then, the derivatives of the complex force resultant with respect to $s$ is

$\frac{d \tilde{T}}{d s}=-\frac{E h}{R_{2}} \vartheta-i \frac{N_{1}}{c}$

wherein $E$ is Young's modulus of shell material; $N_{1}$ is transverse force resultant per unit length on the second principal coordinates; $\vartheta$ is rotate of tangent line of meridian around the second principal coordinate.

\subsection{General solutions for spherical shells and toroidal shells} respectively, expressed in terms of the "complex force resultant"

Three-center-combined shells are composed of a spherical shell and a toroidal shell. The general solutions for both the spherical shells and the toroidal shells subjected to a uniform internal pressure $p$ can be obtained by solving Eqn(3).

The two solutions are expressed in terms of the complex force resultant $\tilde{T}$, in which the general solution for spherical shells is 
$\tilde{T}_{S}=\tilde{B} e^{\beta(1-i)\left(\theta_{0}-\theta\right)}-p R$

$\frac{d \tilde{T}_{s}}{d s}=-\frac{1}{R} \frac{d \tilde{T}_{s}}{d \theta}=\frac{\beta(1-i)}{R} \tilde{B} e^{\beta(1-i)\left(\theta_{0}-\theta\right)}$

where the subscript $S$ indicates the spherical shells, and

$\beta=\sqrt[4]{3\left(1-v^{2}\right)} \sqrt{\frac{R}{h}}$

where $R$ is radius of the spherical shells, and $\tilde{B}$ is a complex constant to be determined.

By assuming

$\alpha=r_{0} / R_{0}$

and

$2 \varepsilon^{2}=\sqrt{12\left(1-v^{2}\right)} r_{0}^{2} /\left(R_{0} h\right)$ (see Fig1 for the definition of $r_{0}$ and $R_{0}$ ), the general solutions and its derivatives for toroidal shells can be expressed as

$$
\begin{gathered}
\tilde{T}_{T}=\frac{i}{2 \varepsilon^{2}} \frac{1}{1+\alpha \sin \theta} \frac{d V}{d \theta}+\frac{3+2 \alpha \sin \theta}{1+\alpha \sin \theta} \frac{p r_{0}}{2} \\
\frac{d \tilde{T}_{T}}{d s}=\frac{1}{r_{0}} \frac{d \tilde{T}_{T}}{d \theta}=\frac{1}{r_{0}}\left[\frac{\sin \theta}{(1+\alpha \sin \theta)^{2}} V-i \frac{2 \varepsilon^{2} \cos \theta}{(1+\alpha \sin \theta)^{2}} A\right]
\end{gathered}
$$

where subscript $T$ indicates the toroidal shells, and

$$
\begin{gathered}
V=(1+\alpha \sin \theta)^{\frac{3}{4}}\left[\tilde{C}_{1} V_{1}+\tilde{C}_{2} V_{2}\right]+\rho \\
\frac{d V}{d \theta}=(1+\alpha \sin \theta)^{\frac{3}{4}}\left[\tilde{C}_{1} \frac{d V_{1}}{d \theta_{1}}+\tilde{C}_{2} \frac{d V_{2}}{d \theta}\right]-\frac{\frac{3}{4} \alpha \cos \theta}{(1+\alpha \sin \theta)^{\frac{1}{4}}}\left(\tilde{C}_{1} V_{1}+\tilde{C}_{2} V_{2}\right)+\frac{d \rho}{d \theta}
\end{gathered}
$$

$A=\frac{1}{\alpha} p\left[\frac{r_{0}}{2} \frac{2+\alpha \sin \theta}{1+\alpha \sin \theta}-\frac{r}{2 \sin \theta}\right](1+\alpha \sin \theta) \sin \theta$

As for the expressions of $V_{1}, V_{2}$ and particular solution $\rho$ in $\operatorname{Eqn}(9)$, see $\operatorname{Eqn}(\mathrm{A} .1)$, (A.3) and (A.5) in appendix for detail.

It is easily seen in Fig. 3 that in the symmetrical plane the joint of the spherical shells and the toroidal shells is such a point that $\theta=\theta_{0}$. Connection conditions on the joint require that their respective horizontal displacement components $\Delta_{x}$, rotations of meridian $\vartheta$, horizontal complex forces $Q_{x}$ and bending moments $M_{1}$ in the symmetric plane are constrained to be equal, that is

$$
\begin{aligned}
& \left.\Delta_{x}\right|_{S}=\left.\left.\Delta_{x}\right|_{T} \quad \vartheta\right|_{S}=\left.\vartheta\right|_{T} \quad\left(\theta=\theta_{0}\right) \\
& \left.Q_{x}\right|_{S}=\left.\left.Q_{x}\right|_{T} \quad M_{1}\right|_{S}=\left.M_{1}\right|_{T} \quad
\end{aligned}
$$

Moreover, since $\Delta_{x}, \vartheta, Q_{x}$ and $M_{1}$ can be expressed in terms of the complex force resultants $\tilde{T}$ and its derivatives, the above 4 connection conditions can be replaced in terms of 2 complex equations as follows:

$\tilde{T}_{S}=\tilde{T}_{T}, \quad \frac{d \tilde{T}_{S}}{d s}=\frac{d \tilde{T}_{T}}{d s} \quad\left(\theta=\theta_{0}\right)$

The complex Eqn(12) are the final connection conditions.

Then let $\theta=\theta_{0}$ in the general solutions (6) and (8), and substitute them into the connection conditions (12), two complex algebraic equations are obtained. Because there are 3 unknown complex constants $\tilde{B}, \tilde{C}_{1}$ and $\tilde{C}_{2}$ in the two equations, a symmetric condition has to be added, which is $d \tilde{T} / d s=0$ when $\theta=\pi / 2$. Thus, the 3 complex constants are uniquely determined by the 3 complex algebraic equations. Then the complex force resultants $\tilde{T}_{S}$ in the spherical shells and $\tilde{T}_{T}$ in the toroidal shells can be determined. Finally, the stress distribution in the three-center-combined shells can be determined by using Eqn(A.8) and (A.9).

\section{Numerical results based on the combined shells model and a comparison with $A B A Q U S$ results}

Stress distributions in a red blood cell subjected to an osmotic pressure are calculated in two different ways. The first way is the use of the previous analytical Eqn(6) and (8) based on the combined shells model. The second way is the use of ABAQUS software according to the actual size of cells. Calculation data are cited from the papers by Fung [4], Dao [13], Zarda, et al. [7] and Skalak, et al. [14], respectively. Wherein the modulus of elasticity of the cell membrane is $E=2.1 \times 10^{-9} \mathrm{~N} / \mu \mathrm{m}^{2}$; Poisson's ratio $v=0.3$; membrane thickness $h=0.1 \mu \mathrm{m}$; Osmotic pressure $P=1 \times 10^{-14} \mathrm{~N} / \mu \mathrm{m}^{2}$.

When the combined shells model is used, the analytical results are obtained by substituting above data into the previous Eqn(6) and (8) which are programmed in MATLAB. Finally, the radial stress $\sigma_{1}$ and the annular stress $\sigma_{2}$ are $\sigma_{1}=\frac{6 M_{1}}{h^{2}}, \quad \sigma_{2}=\frac{T_{2}}{h}$

where $M_{1}$ is the moment resultants per unit length in radial direction, $T_{2}$ is the force resultants per unit length in annular direction. Results are depicted in Fig. 5. Wherein the abscissa represents the horizontal distance in the cell (see Fig. 3), the ordinate in Fig. 5(a) represents the ratio of the radial stress by mean stress, $\sigma_{1}: p r / h$, and the ordinate in Fig. 5(b) represents the ratio of the annular stress by mean stress, $\sigma_{2}: p r / h$.

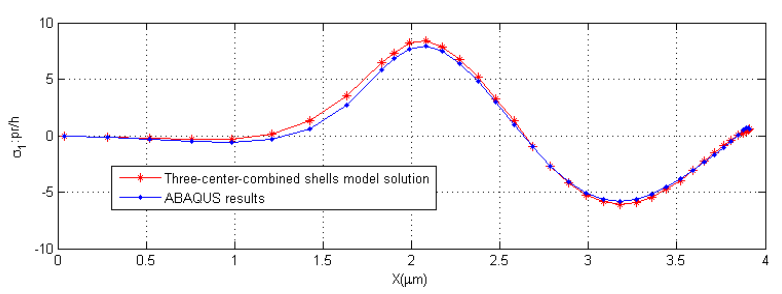

(a) ratio of the radial stress by mean stress

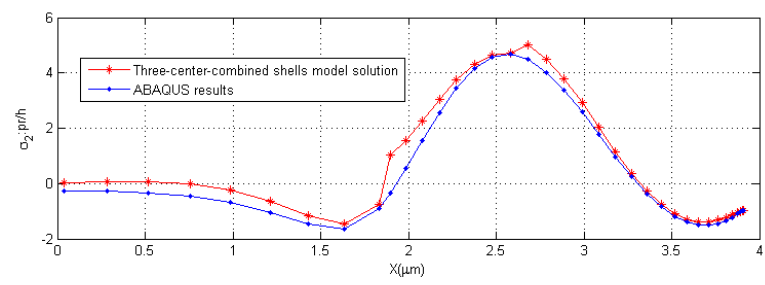

(b) ratio of the annular stress by mean stress

Fig. 5 Comparison of three-center-combined shells solution and ABAQUS results

It is seen in Fig. 5(a) that the analytical solution based on the three-center-combined shells model gives a close agreement with the ABAQUS numerical results. It is also seen in Fig. 5(a) that the bending stress $\sigma_{1}$ reaches its maximum value in the vicinity of joint line of spherical shells and toroidal shells. It is apparently because, as mentioned before, the Gaussian curvature changes its sign on the joint line from positive to negative. Therefore, cell membranes have the capacity to sustain moment, and behave as a "real membrane". It is worthy to note that above argument is viewed as a hypothesis in the papers by Tao [1], Zarda, et al. [7] and Evans 
[15], respectively. It is seen in Fig. 5(b) that the annular stress $\sigma_{2}$ reaches its maximum value in the portion of toroidal shells. Finally, it can be concluded that all stress components (including $\sigma_{1}$ and $\sigma_{2}$ ) in the portion of toroidal shells are fluctuant and higher in comparison with those in the portion of spherical shells.

\section{Conclusions}

In the present paper a new model of three-center-combined shells is proposed to simulate the geometry of red blood cells. Based on the model, the stress distribution in the membrane is calculated in the case of small deformation by using measured data in osmosis experiments. The following conclusions can be summarized as follows:

1) The three-center-combined shells model can well simulate the biconcave shape of red blood cells. Using the model, the surface area and volume, the stress distribution in the membrane of red blood cells can be conveniently calculated. The obtained results are good agreement with experimental results or numerical results.

2) There exist bending moments in the vicinity of joint line between spherical and toroidal areas on the membrane of biconcave red blood cells, where the Gaussian curvature changes sign from positive to negative. In this case, the membrane of red blood cells has the capacity to sustain bending moments and behaves as a shell.

\section{ACKNOWLEDGEMENTS}

Supporting by the China National Science Foundation (Grant No. 11072178 and 11172214) is gratefully acknowledged.

\section{References}

[1] Tao, Z., Biological fluid dynamics, Beijing: Science Press, 1984.

[2] Libai, A., Nonlinear Membrane Theory. in Theoretical and applied mechanics 1992, edited by Bodner,S.R., et al., Proceedings of the XVIIIth International Congress of Theoretical and Applied Mechanics, Haifa, Israel, 22-28 August 1992.

[3] Beck, J.S., Relations between membrane monolayers in some red cell shape transformations. J Theor Biol 75 (4), 1978, 487-501.

[4] Fung, Y.C., Biomechanics: mechanical properties of living tissues, New York, Springer, 12 (1993).

[5] Evans, E.A., A new material concept for the red cell membrane, Biophysical journal 13 (9), 1973, 926-940.

[6] Helfrich, W. and Deuling H.J., Some theoretical shapes of red blood cells, J. Phys., 36 (1975), 327-329.

[7] Zarda, P.R., Chien S. and Skalak R., Elastic deformations of red blood cells, Journal of Biomechanics, 10(4), 1997, 211-221.

[8] Zhan, Q., Zhang R.J. and Jiao, G., Deformation analysis of red blood cells in osmosis experiment, Journal of medical biomechanics, 27(6), 2012, 687-691.

[9] Novozhilov, V.V., Thin shell theory, P. Noordhoff, 25 (1959).

[10] Evans, E. and Fung Y.C., Improved measurements of the erythrocyte geometry, Microvascular research 4 (4), 1972, 335-347.

[11]Tsang, W., The size and shape of human red blood cells, MS thesis, University of California at San Diego, San Diego, Calif., 1975.

[12] Angelov, B. and Mladenov, I. M.,On the Geometry of Red Blood Cell,In: Geometry, Integrability and Quantization 1,2000, 27-46

[13]Dao, M., Lim, C.T. and Suresh, S., Mechanics of the human red blood cell deformed by optical tweezers, Journal of the Mechanics and Physics of Solids, 51(11-12), 2003, 2259-2280.

[14]Skalak, R., et al., Strain energy function of red blood cell membranes,
Biophysical journal 13(3), 1973, 245-264.

[15]Evans, E., Bending resistance and chemically induced moments in membrane bilayers, Biophysical journal 14(12), 1974, 923-931.

\section{Appendix}

1. Function $V_{1}$ and $V_{2}$ are determined by the following formulas:

If $\theta$ is positive

$$
\begin{aligned}
& V_{1}(\sqrt{2 i} \xi)=\frac{1}{2^{\frac{1}{12}} \xi^{\frac{1}{6}}} e^{-\xi}\left[\cos \left(\xi-\frac{\pi}{24}\right)+i \sin \left(\xi-\frac{\pi}{24}\right)\right] \\
& V_{2}(\sqrt{2 i} \xi)=\frac{1}{2^{\frac{1}{12}} \xi^{\frac{1}{6}}} e^{\xi}\left[\cos \left(\xi+\frac{\pi}{24}\right)-i \sin \left(\xi+\frac{\pi}{24}\right)\right]
\end{aligned}
$$

where

$$
\xi=\varepsilon \int_{0}^{\theta} \frac{\sqrt{\sin \theta}}{\sqrt{1+\alpha \sin \theta}} d \theta=\varepsilon \int_{0}^{\frac{\pi}{2}} \frac{\sqrt{\sin \theta}}{\sqrt{1+\alpha \sin \theta}} d \theta
$$

If $\theta$ is negative

$$
\begin{aligned}
& \bar{V}_{1}(\sqrt{2 i} \xi)=\frac{1}{2^{\frac{1}{12}} \xi^{\frac{1}{6}}} e^{-\zeta}\left[\cos \left(\zeta-\frac{\pi}{24}\right)-i \sin \left(\zeta-\frac{\pi}{24}\right)\right] \\
& \bar{V}_{2}(\sqrt{2 i} \xi)=\frac{1}{2^{\frac{1}{12}} \xi^{\frac{1}{6}}} e^{\zeta}\left[\cos \left(\zeta+\frac{\pi}{24}\right)+i \sin \left(\zeta+\frac{\pi}{24}\right)\right]
\end{aligned}
$$

where

$$
\zeta=\varepsilon \int_{0}^{\varphi} \frac{\sqrt{\sin \varphi}}{\sqrt{1-\alpha \sin \varphi}} d \varphi=\varepsilon \int_{0}^{\theta_{0}} \frac{\sqrt{\sin \varphi}}{\sqrt{1-\alpha \sin \varphi}} d \varphi(\mathrm{A} .17)
$$

2. Function $\rho$ is determined by the following formula:

$$
\rho=a_{1} \cos \theta+b_{2} \sin 2 \theta+a_{3} \cos 3 \theta+b_{3} \sin 4 \theta+\cdots \text { (A.18) }
$$

Where $a_{j}, b_{j}$ are

$$
\frac{a_{n}}{b_{n-1}} i=\frac{1+i \frac{(n-1)(n-2)}{2 \varepsilon^{2}} \alpha}{\frac{n^{2}}{\varepsilon^{2}}+\frac{\left[1+i \frac{n(n-1)}{2 \varepsilon^{2}} \alpha\right]\left[1+i \frac{(n+1)(n+2)}{2 \varepsilon^{2}} \alpha\right]}{\frac{(n+1)^{2}}{\varepsilon^{2}}+\frac{\left[1+i \frac{(n+1) n}{2 \varepsilon^{2}} \alpha\right]\left[1+i \frac{(n+2)(n+3)}{2 \varepsilon^{2}} \alpha\right]}{\frac{(n+2)^{2}}{\varepsilon^{2}}+\cdots}}}
$$

$$
-\frac{b_{n+1}}{a_{n}} i=\frac{1+i \frac{n(n-1)}{2 \varepsilon^{2}} \alpha}{\frac{(n+1)^{2}}{\varepsilon^{2}}+\frac{\left[1+i \frac{(n+1) n}{2 \varepsilon^{2}} \alpha\right]\left[1+i \frac{(n+2)(n+3)}{2 \varepsilon^{2}} \alpha\right]}{\frac{(n+2)^{2}}{\varepsilon^{2}}+\frac{\left[1+i \frac{(n+2)(n+1)}{2 \varepsilon^{2}} \alpha\right]\left[1+i \frac{(n+3)(n+4)}{2 \varepsilon^{2}} \alpha\right]}{\frac{(n+3)^{2}}{\varepsilon^{2}}+\cdots}}}
$$

3. Hoop tension $T_{2}$ and radical moment $M_{1}$ of spherical 